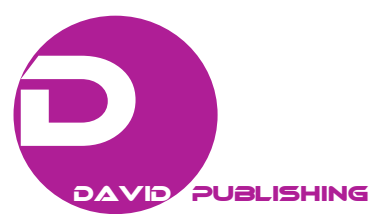

\title{
Study of Changes in the Thermodynamic Functions in a
}

\section{Magnetic Field}

\author{
Aibassov Yerkin Zhakenovich ${ }^{*}$, Yemelyanova Valentina, Tussupbayev Nessipbay, Shakieva Tatyana and \\ Yerzhanova Zhadyra \\ Research Institute of New Chemical Technologies and Materials, Kazakh National University Al-Farabi, Almaty 005012, Kazakhstan
}

Abstract: The authors offered the new formula of the thermodynamic functions in a magnetic field. The authors have also found that thermodynamic functions of internal energy $d U$ and free energy $d F$ changes in the magnetic field.

Key words: Thermodynamic functions, magnetic field, internal and free energy.

\section{Introduction}

In recent years, a large number of works have devoted to the influence of magnetic field on the thermodynamic functions.

The aim of the work is how to behave inside $d U$ and free energy $d F$ under the action of a magnetic field.

\section{Theory}

The internal energy $U$ is determined according to the first law of thermodynamics, as the difference between the amount of heat, system messages, and the work done by the system on external bodies:

$$
U=Q-A
$$

Helmholtz free energy $F$ is defined as follows:

$$
F=U-T S
$$

where, $T$-temperature and $S$-entropy.

Since the isothermal process the amount of heat received by the system, as well $T \Delta S$, then loss of free energy in the quasi isothermal process is working, committed system on external bodies.

Enthalpy $H$ - the thermodynamic potential of the state of the system is in thermodynamic equilibrium in the selection of the independent variables as pressure,

\footnotetext{
*Corresponding author: Aibassov Yerkin Zhakenovich, professor, research fields: organic chemistry of $\mathrm{U}, \mathrm{Th}, \mathrm{As}, \mathrm{Sb}$, Bi. E-mail: erkin53@mail.ru.
}

entropy, and the number of particles.

Enthalpy or energy of the extended system $E$ is the sum of the internal energy of the gas $U$ and the potential energy of the piston with the load $E_{\text {potent }}=$ $P S x=P V:$

$$
H=E=U+P V
$$

Enthalpy is the sum of the internal energy of the body and the work that must be expended to enter the body of volume $V$ in an environment having a pressure $P$ and located the body in equilibrium. Enthalpy $H$ is similar to the internal energy and other thermodynamic potentials have a definite value for each state, which is a function of the state [1-12].

$$
\Delta H=H_{2}-H_{1}
$$

Changes Enthalpy does not depend on the way the process, it is defined only the initial and final state of the system. If the system in any way be returned to its original state (cyclic process), then change any of its parameters is a function of the state is zero, hence $\Delta H$ $=0$.

In the construction of relativistic thermodynamics (taking into account the special theory of relativity), a convenient approach is to use the so-called invariant enthalpy.

In this approach, the temperature $T$ is defined as the Lorentz-invariant. Entropy $S$ is also invariant. Since the walls of the system affect the independent variable is the pressure $P$, and therefore the 
thermodynamic potential as it is convenient to take enthalpy.

For such a system enthalpy and momentum of the system $g$ form a 4-vector, and for defining the invariant enthalpy the same in all frames of reference, taken invariant function of the 4 -vector:

$$
H=\sqrt{(U+P V)^{2}}-c^{2} g^{2}
$$

The basic equation of relativistic thermodynamics is recorded through the differential invariant enthalpy as follows:

$$
d H=T d s+V / \sqrt{1-v^{2} / c^{2}} d P+\mu d N
$$

Using this equation, the authors can solve many problems thermodynamics moving systems, if the function $H(S, P, N)$.

In view of the magnetic field, this equation will be expressed as follows:

$$
d H=T d s+V / \sqrt{1-v^{2} / c^{2}} d P+\mu d N+1 / 4 \pi H d B
$$

\section{Results and Discussion}

Consider the effect of a magnetic field on the internal energy $d U$ and free energy $d F$. In an external magnetic field, it is given by the vector magnetic induction $B$ and changes the values of the thermodynamic potentials of thermodynamic systems. For example, the increase in internal energy per unit volume of a thermodynamic system to the extent that the magnetic field is equal to the $d B[13,14]$ :

$$
d U=T d S+1 / 4 \pi H d B
$$

where, $S$-entropy, $T$-temperature.

Accordingly, for the free energy $d F$, the authors obtain the Eq. (9):

$$
d F=-S d T+1 / 4 \pi H d B
$$

\section{Conclusions}

Thus, the authors have found how thermodynamic functions internal energy $d U$ and free energy $d F$ change in the magnetic field, it have the following forms:

$$
\begin{gathered}
d U=T d S+1 / 4 \pi H d B \\
d F=-S d T+1 / 4 \pi H d B
\end{gathered}
$$

The authors assume that a similar dependence on the magnetic field will have an entropy $H$ and enthalpy $S$.

\section{References}

[1] Ilya Prigogine, I., and Defay, R. 1954. Chemical Thermodynamics. Translated by Everett, D. H. London: Longmans, Green \& Co.

[2] Enrico, F. 1956. Thermodynamics. New York: Courier Dover Publications.

[3] Helmholtz, H. V. 1897. Lectures on Theory of Heat, edited by Richarz, F. Leipzig: Press of Johann Ambrosius Barth, Section 46, pp. 176-82.

[4] Clausius, R. 1850. "About Moving Power of the Heat and the Laws Which Can Be Derived from It for Thermodynamics." Annals of Physics and Chemistry 155 (3): 368-94.

[5] Bridgman, P. W. 1943. The Nature of Thermodynamics. Cambridge MA: Harvard University Press, pp. 48.

[6] Partington, J. R. 1913. A Text-book of Thermodynamics. New York: Van Nostrand, pp. 37.

[7] Glansdorff, P., and Prigogine, I. 1971. Thermodynamic Theory of Structure, Stability and Fluctuations. London: Wiley-Interscience, pp. 15.

[8] Buchdahl, H. A. 1966. The Concepts of Classical Thermodynamics. London: Cambridge University Press, pp. 117-8.

[9] Hess, H. 1840. "Thermochemische Untersuchungen." Annals of Physics and Chemistry 126 (6): 385-404.

[10] Lewis, G. N., and Randall, M. 1923. Thermodynamics and the Free Energy of Chemical Substances. New York: McGraw-Hill Book Co. Inc.

[11] Guggenheim, E. A. 1933. Modern Thermodynamics by the Methods of J.W. Gibbs. London: Methuen.

[12] Glansdorff, P., and Prigogine, I. 1971. Thermodynamic Theory of Structure, Stability and Fluctuations. London: Wiley-Interscience, pp. 15.

[13] Aibassov, Y. Z., and Yemelyanova, V. S. 2015. Spin Chemistry and Magnetic of Uranium-Thorium Catalysts. New York: Scientific \& Academic Publishing, pp. 232.

[14] Aibassov, Y. Z., and Yemelyanova, V. S. 2015. Spin Catalytic Reaction of NO and New Reactions of Arsenic, Antimony and Bismuth. New York: Scientific \& Academic Publishing, pp. 328. 